\title{
Forensic Geotechnical Engineering Challenges
}

\author{
Dr. Ahmed Fahmy \\ Senior Geotechnical Engineer at AECOM, \\ New Jersey, USA
}

Recent years have witnessed a surge in geotechnical related damage and, therefore, an increased number of geotechnical related legal and insurance claims.

During this presentation, an overview of the forensic geotechnical engineering science will be first given, along with a discussion on how to apply engineering design principles to help answer questions that could have legal implications on the involved parties in the investigated project.

This will be followed by a review of a number of notable recent statistics, giving an insight on the geotechnical related failures in the last few years in North America.

An in-depth discussion of the non-engineering aspects of geotechnical forensic investigations will be then provided. This includes the typical clients in the Forensic Engineering business, and how different their mindset is from engineers. As well, a thorough discussion of the technical and ethical challenges a forensic engineer typically faces in any investigation he/she gets involved in will be given.

Finally, a number of Canadian and international case studies will be discussed, along with a review of the latest investigation techniques adopted in this sector. For each of the cases, a thorough review of the origin and cause of the reported damage and a commentary on the standard of care of geotechnical engineer(s) will be provided, along with the applied remediation measures to fix the affected structures. 